(1)

CrossMark

\title{
COPD biomarkers and phenotypes: opportunities for better outcomes with precision imaging
}

\author{
George R. Washko ${ }^{1}$ and Grace Parraga ${ }^{2,3}$
}

Number 2 in the series "Controversies in COPD: What Can be Done to Move the Field Forward?"

Edited by D.D. Sin

Affiliations: ${ }^{1}$ Brigham and Women's Hospital, Harvard Medical School, Boston, MA, USA. ${ }^{2}$ Robarts Research Institute, Western University, London, ON, Canada. ${ }^{3}$ Dept of Medical Biophysics, Western University, London, ON, Canada.

Correspondence: G. Parraga, Robarts Research Institute, 1151 Richmond Street North, London, ON, Canada N6A 5B7. E-mail: gparragađrobarts.ca

@ERSpublications

COPD imaging has not improved outcomes in patients with advanced lung disease. For patients to benefit, imaging phenotypes must be developed that reflect early, reversible disease or be used in a more pragmatic fashion to improve clinical outcomes. http://ow.ly/i19g30m7f2I

Cite this article as: Washko GR, Parraga G. COPD biomarkers and phenotypes: opportunities for better outcomes with precision imaging. Eur Respir J 2018; 52: 1801570 [https://doi.org/10.1183/13993003.015702018].

ABSTRACT A number of chronic diseases have benefited from both imaging and personalised medicine, but unfortunately, for patients with chronic obstructive pulmonary disease (COPD), there has been little clinical uptake or recognition of the key advances in thoracic imaging that might help detect disease early, or, perhaps more importantly, might help develop and phenotype patients for novel or personalised therapies that may halt disease progression. We outline our vision for how computed tomography and magnetic resonance imaging may be used to better inform COPD patient care, and, perhaps more importantly, how these may be used to help develop new therapies directed at early disease. We think that imaging and precision medicine should be considered and used together as "precision imaging" at specific stages of COPD when the major pathologies may be more responsive to therapy. While "precision medicine" is the tailoring of medical treatment to individual patients, we define "precision imaging" as the tailoring of specific therapies and interventions to individual patients with a detailed quantitative understanding of their specific imaging phenotypes and measurements. Finally, we stress the importance of "seeing" the pathology, because without this understanding, you can neither treat nor cure patients with COPD.

Previous articles in this series: No. 1: Kim V, Aaron SD. What is a COPD exacerbation? Current definitions, pitfalls, challenges and opportunities for improvement. Eur Respir J 2018; 52: 1801261.

Received: Aug 172018 | Accepted after revision: Sept 272018

Copyright @ERS 2018 


\section{Introduction}

Precision medicine offers the hope for therapy that is tailored to individual patients and results in minimised therapeutic risk and improved clinical outcomes. This is particularly relevant for patients with chronic obstructive pulmonary disease (COPD) where there is marked heterogeneity in both the structural and clinical manifestations of disease as well as therapeutic outcomes. For example, it is well understood that individual COPD patients with similar degrees of spirometric impairment can be very different in terms of symptoms, exercise capacity and exacerbation risk. Moreover, these same patients may also have marked differences in the extent of parenchymal damage (or emphysema) and airway abnormalities, including airway wall remodelling, mucous plugging and airway obliteration. Finally, the pathological determinants of COPD often reside in the silent zones of the peripheral lung where, because of its redundant design in peak health, they can progress undetected or are incorrectly ascribed to advancing age, until disease is quite advanced. Thoracic imaging provides tools that enable the in vivo detection of these processes and we believe that the inclusion of such data in therapeutic decision making, i.e. "precision imaging", will improve patient care. While precision medicine refers to the tailoring of treatment to the physiology of individual patients, precision imaging is a way to tailor specific therapies and interventions to patients based on their imaging phenotypes and measurements. This goal is not new, but we believe that past efforts have been incomplete and, in many cases, not sufficiently bold.

There have been innumerable reports on the application of imaging in COPD patients, but aside from efforts to mechanically deflate emphysematous lung, they have had little impact on patient care, leaving many clinicians thinking that it is unnecessary (at best) and resource-wasteful (as worst) to requisition more than a chest radiograph for clinical decision making. Some of the reasons for this include a limited ability to deploy advanced imaging methodologies at the site of clinical care, an absence of emphasis on the detection of "early" disease and, finally, a failure to recognise that while not every patient with advanced disease may benefit from imaging, there are subgroups in which such efforts may afford a marked clinical benefit.

Our intention is to provide guidance on how imaging can be used to enhance patient care. To do this we will begin by outlining a broad set of unmet needs in clinical care followed by a brief review of advances in computed tomography (CT) and magnetic resonance imaging (MRI) (table 1). Finally, we provide some forward-looking suggestions on the application of imaging in the detection of early disease and the management of more advanced COPD (table 2). This is not meant to be a comprehensive review of imaging or clinical care, but rather an opinion piece that stimulates dialogue and constructive critique.

\section{The goals of imaging for COPD patients and at-risk ex-smokers}

Smoking cessation is one of the most impactful interventions in clinical care. It slows the decline in lung function and improves survival $[1,2]$. Additional treatments such as oxygen therapy and lung volume reduction surgery have also been shown to improve survival in select patients [3-5], but the additional benefits afforded by pharmacological therapy have been more modest. These latter treatments may

TABLE 1 State-of-the-art computed tomography (CT) and magnetic resonance imaging (MRI) measurements for chronic obstructive pulmonary disease phenotyping

$\begin{array}{lll}\text { Phenotypes CT MRI } & \end{array}$

\begin{tabular}{lcc}
\hline Airway wall thickness & Wall area thickness, Pi10 & $x$ \\
Airway lumen calibre & Lumen area & $x$ \\
Ventilation abnormalities & Ventilation & Ventilation defect percent \\
Small vessels & $\checkmark$ & $x$ \\
Perfusion & $\checkmark$ & $\checkmark$ \\
Large arteries & $\checkmark$ & $\checkmark$ \\
Gas trapping & $<-856 \mathrm{HU}$; PRM for functional small airways & $\checkmark$ \\
Emphysema & $<-950$ HU; PRM for emphysema & Apparent diffusion coefficients \\
Bronchiectasis & $\checkmark$ & Airway filling \\
Fibrosis & $\checkmark$ & $\checkmark$ \\
Pulmonary artery & Pulmonary artery/aorta ratio & $x$ \\
$\quad$ abnormalities & & $\checkmark$
\end{tabular}

$\boldsymbol{X}$ : not yet observed or studied. Pi10: square root wall area for airway with internal perimeter of $10 \mathrm{~mm}$; PRM: parametric response mapping. 
TABLE 2 Recommendations on optimal use of computed tomography (CT) and magnetic resonance imaging (MRI) to improve chronic obstructive pulmonary disease outcomes

\begin{tabular}{|c|c|c|c|c|c|c|}
\hline & \multicolumn{3}{|c|}{ СT (structure) } & \multicolumn{3}{|c|}{ MRI (structure-function) } \\
\hline & Airways & Parenchyma & Vessels & Airways & Parenchyma & Vessels \\
\hline Disease detection & $\checkmark$ & $\checkmark$ & $\checkmark$ & $\checkmark$ & $\checkmark$ & $x$ \\
\hline Early disease phenotyping & $x$ & $\checkmark$ & $x$ & $\checkmark$ & $\checkmark$ & $x$ \\
\hline Image-guided therapy & $x$ & $\checkmark$ & $x$ & $\checkmark$ & $x$ & $x$ \\
\hline Measure/monitor treatment response & $x$ & $x$ & $x$ & $\checkmark$ & $x$ & $x$ \\
\hline Predict exacerbation & $x$ & $x$ & $x$ & $\checkmark$ & $\checkmark$ & $x$ \\
\hline
\end{tabular}

$\boldsymbol{X}$ : not yet observed or studied.

improve lung function, and reduce COPD symptoms [6], exacerbations and hospitalisations [7, 8], but their effect is not large and generally not discernible on a per-patient basis. Furthermore, COPD hospitalisation and mortality rates continue to rise, especially in females [9]. In light of this, how can imaging methods and measurements be strategically used in COPD patients?

Based on our collective experience in cohort and small patient studies, we think efforts must be based upon an understanding of disease pathobiology and how these processes change with disease progression. For example, imaging measurements of therapy response offered at a certain stage of disease may be unhelpful, yet, if therapy had been offered at an earlier time, the disease trajectory may have been directly measured and altered. Figure 1 shows histological evidence of different disease stages starting in the acinus with inflammation (figure 1b), remodelling (figure 1c), and destruction of the distal airway and parenchyma (figure 1d) [10]. This evidence supports the concept that while an anti-inflammatory therapy may be highly effective in patients with inflammatory abnormalities, this is unlikely to be the case for airway wall remodelling and certainly not in severe emphysematous destruction when the benefits likely do not outweigh the risks and cost. These histological findings, described over six decades ago [10], clearly show that without "seeing" the pathology responsible for symptoms and disease worsening, you cannot treat it and you certainly cannot cure it.

Precision imaging in COPD patients should also go beyond efforts to measure and arrest disease progression, and should focus on optimising therapies that reduce hospitalisation and prevent re-hospitalisation. This further emphasises the time dependence of our model of precision imaging and how this needs to incorporate susceptibility to events such as acute respiratory exacerbations and their response to treatment. Imaging can be a foundation for such a holistic vision, but it is clear that such efforts will have to incorporate additional biomarkers as well as clinical data and be constantly in flux based upon the patient experience. To guide these efforts, precision imaging should focus on the three

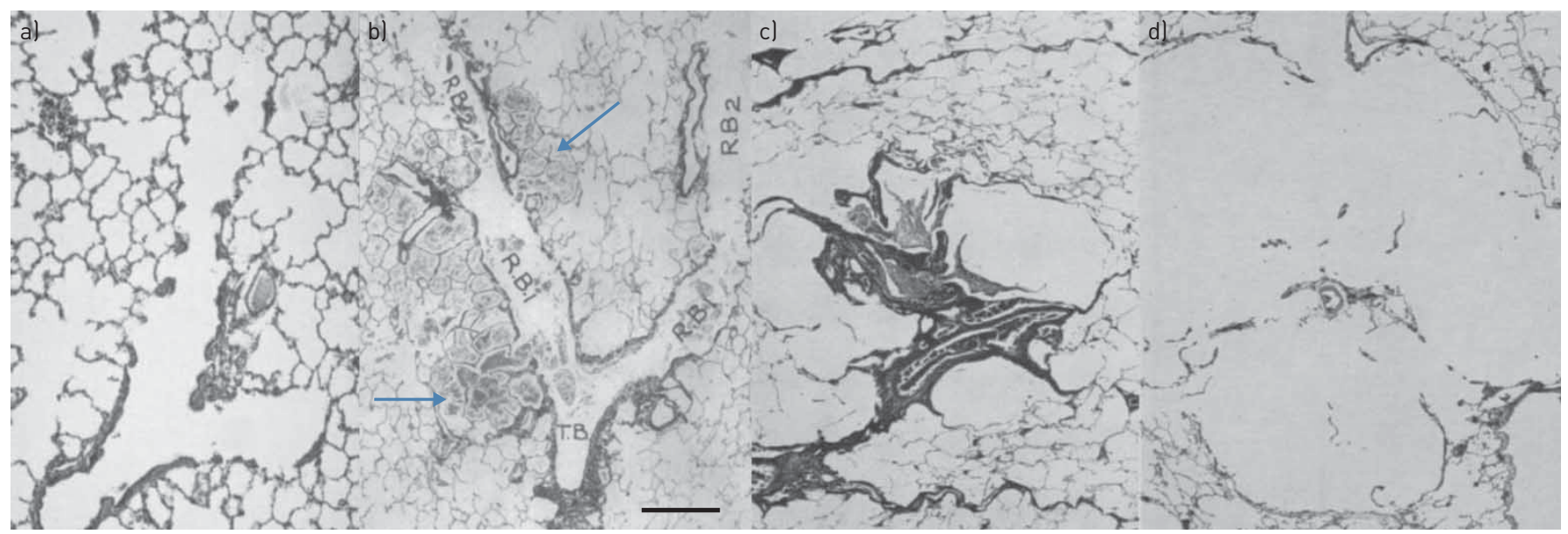

FIGURE 1 Histological evidence of airway and alveolar abnormalities across the spectrum of chronic obstructive pulmonary disease severity. a) Normal alveolar pathology. b) Airway inflammatory abnormalities (arrows). RB1 and 2: orders of respiratory bronchioles; TB: terminal bronchiole. c) Airway wall remodelling. d) Severe emphysematous destruction. Scale bar: $50 \mu \mathrm{m}$. Reproduced and modified from [10] with permission. 
general compartments of the lung: 1) the parenchyma, including the terminal bronchioles, alveoli and the alveolar gas-blood tissue barrier, 2) the airway tree, including the large and small airways, and 3) the large pulmonary vessels and the small vessel tree.

For the purposes of this perspective we focus on advances pertinent to thoracic CT where the bulk of research experience lies and then we review emerging functional MRI biomarkers of COPD developed over the past few years. The main contributions of CT thus far include high-resolution emphysema, vessel and airway measurements to the fifth and seventh generation, close to the small airways, while the main contributions of MRI relate to functional and serial measurements of ventilation abnormalities and parenchyma microstructure in early subclinical disease.

\section{Computed tomography \\ Emphysema}

Regional tissue destruction or emphysema is very common in COPD patients; in full-inspiration CT images this appears as regions of low attenuation because of the loss of tissue and increased presence of air. CT densitometry threshold values and percentile points have been extensively used to quantify emphysema, including -950 HU [11-13] and the 15th percentile on the frequency distribution curve [14]. Figure 2 shows CT images with all lung voxels less than the $-950 \mathrm{HU}$ threshold highlighted. The three-dimensional reconstructions of the CT airway tree and the low-attenuation clusters (LAC;
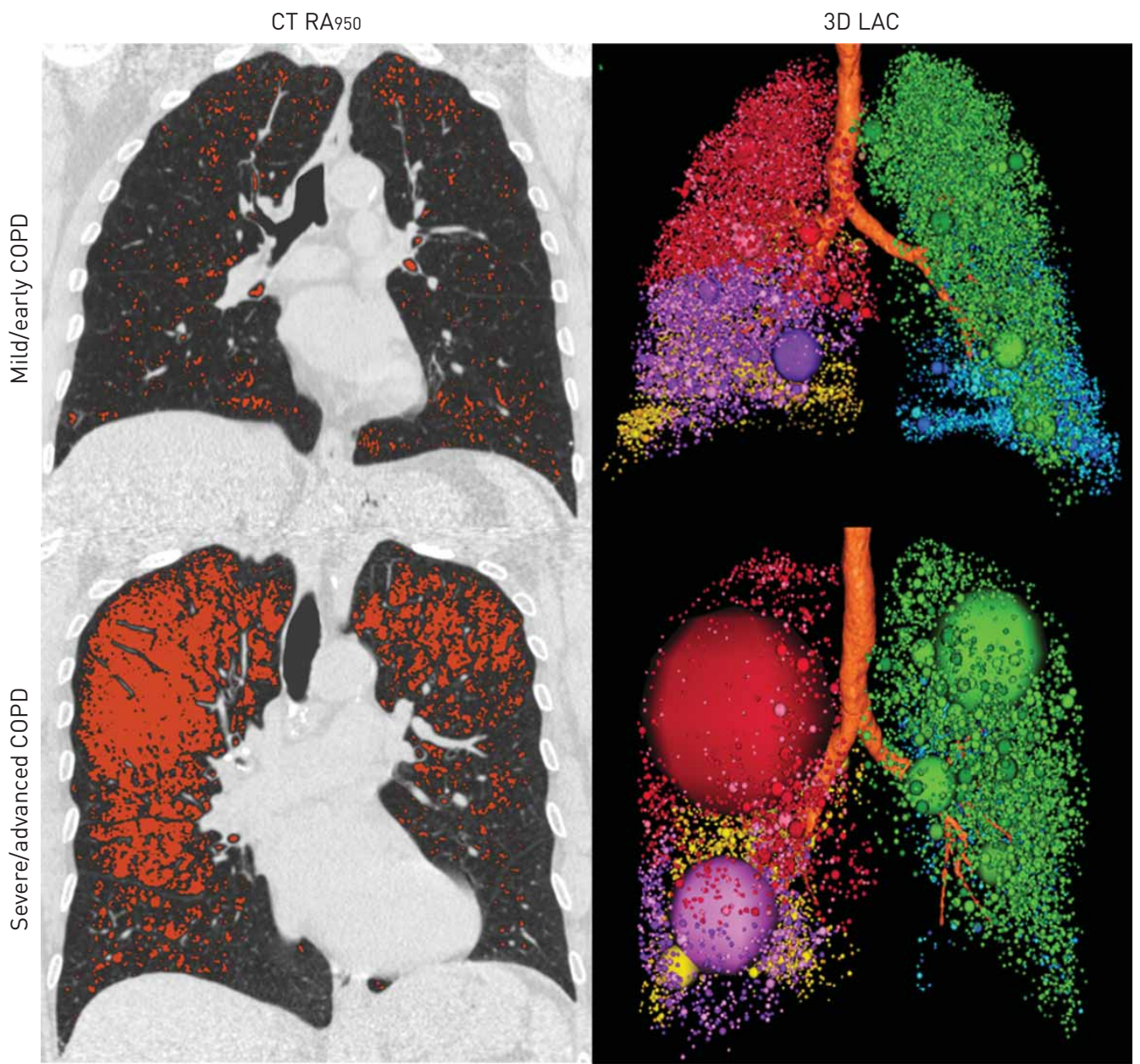

FIGURE 2 Centre coronal thoracic computed tomography (CT) emphysema measurements. RA950: relative area of the CT density histogram voxels < $-950 \mathrm{HU}$; 3D: three-dimensional; LAC: low-attenuation $<<-950 \mathrm{HU}$ ) clusters; COPD: chronic obstructive pulmonary disease; FEV1: forced expiratory volume in $1 \mathrm{~s}$; FVC: forced vital capacity; RV: residual volume; TLC: total lung capacity; DLCO: diffusing capacity of the lung for carbon monoxide; 6MWD: 6-min walk distance. Mild/early COPD: 74-year-old male, FEV1 86\% predicted, FEV1/FVC $59 \%$, RV/TLC 88\% predicted, DLCo 45\% predicted, 6MWD 486 m, pack-years 40, RA950 6\%, LAC -2.0. Severe/ advanced COPD: 71-year-old female, FEV1 47\% predicted, FEV1/FVC 41\%, RV/TLC 144\% predicted, DLCo 31\% predicted, 6MWD $441 \mathrm{~m}$, pack-years 52, RA950 22\%, LAC -1.61 . 
colour-coded for the different lung lobes) are also shown. Although it is clear for the patient with severe/advanced COPD that emphysema is predominantly in the upper and middle lobes, the LAC analysis also shows a visual representation of the size of emphysematous bullae and their location as well as quantitative measurements. Notably, CT emphysema measurements can be used to identify patients that may have greater responses to lung volume reduction surgery and endobronchial valve implantation $[3,15]$.

\section{Airways}

Airways disease, chronic bronchitis and chronic sputum production are also hallmark pathological features of COPD. The proximal airways can be directly visualised and quantified using CT methods, and numerous approaches have been used to segment the airway tree and then quantify the airway wall dimensions with the belief that mural thickening in the central airways represents a distinct pathological process. The results of these efforts have repeatedly demonstrated that objective assessments of central airway morphology provide data that are statistically complementary to parenchymal density measurements in the prediction of lung function. Other work has also demonstrated that airway morphology can predict respiratory exacerbations [16] and the risk of future lung disease [17], and is abnormal in symptomatic smokers with normal spirometry [18]. The critical challenge that remains, however, is the clinical translation of CT airway measurements. The relatively large intersubject variability in these measures and their narrow dynamic range means that while they may provide some insight into trends in large cohorts, they are still of limited clinical utility in individual patients. Another critical limitation stems from the fact that early findings in relation to airway abnormalities are nearly never identified because CT is not used to phenotype ex-smokers without spirometric evidence of disease.

There are also approaches that attempt to quantify small airway disease using expiratory CT imaging. These include simple densitometric thresholding of the expiratory CT scan as well calculation of the inspiratory to expiratory volume change of voxels with attenuation values from -860 to $-950 \mathrm{HU}$ [19], and the expiratory/inspiratory ratio of mean lung density in Hounsfield units [20, 21] and Jacobian analyses [22]. Another approach uses voxel-by-voxel comparison of CT attenuation after co-registration of inspiration and expiration CT, otherwise known as parametric response mapping (PRM) [23, 24]. Such PRM measurements have been shown to be reproducible over short periods of time [25], correlated with pulmonary function, showed spatial agreement with MRI functional abnormalities [26] and were associated with longitudinal changes in forced expiratory volume in $1 \mathrm{~s}$ [27]. There is ongoing work to determine the histopathological basis of these measures and with those efforts, their clinical utilisation may become better understood.

\section{Perfusion and the pulmonary vasculature}

There are several techniques under investigation for the static assessment of the pulmonary vascular tree and heart. These generally provide measurements of blood vessel volume and vascular tortuosity for the intraparenchymal vessels as well as epicardial measures of the right and left ventricular volumes [28-30]. Such techniques have been used for disease stratification and, in smaller studies of arterial and venous segmented vasculature, for discrimination of disease subtypes. Further preliminary work focused on the heart suggests that such techniques may be used to identify patients who may benefit most from therapies to reduce the risk for acute respiratory exacerbations [31].

A limitation to these approaches is that they cannot provide direct assessments of lung ventilation or perfusion, i.e. functional information. Therefore, other approaches such as dual-energy CT (DECT) [32-34] have been developed and evaluated. A recent study investigating DECT with combined xenon-enhanced ventilation and iodine-contrast-enhanced perfusion imaging demonstrated the feasibility of obtaining regional and quantitative ventilation and perfusion measurements and, importantly, the measurements of the ventilation-perfusion relationship on a voxel-by-voxel basis [35]. In patients with COPD, these DECT ventilation-perfusion measurements were shown to be significantly associated with measures of pulmonary function [35].

\section{Magnetic resonance imaging}

Functional and microstructural MRI

Pulmonary MRI has been developed as a research tool in part due to its speed, excellent safety and tolerability profile $[36,37]$, and because of the unique combination of morphological and functional phenotypes MRI provides. Morpho-functional pulmonary measurements can be achieved using conventional proton $\left({ }^{1} \mathrm{H}\right)$ MRI using the inherent signal of the parenchyma. In addition, functional information may also be derived using physiological signal alterations such as pulsatile blood flow or ventilation stemming from the ${ }^{1} \mathrm{H}$ MRI signal itself, or using intravenous or inhaled contrast agents. 
Ventilation and diffusion-weighted inhaled noble gas MRI

MRI with inhaled hyperpolarised helium $\left({ }^{3} \mathrm{He}\right)$ or xenon $\left({ }^{129} \mathrm{Xe}\right)$ gas provides a unique and direct way to measure pulmonary ventilation and the destruction of the terminal bronchi and alveoli [38]. Parenchyma microstructural information can be obtained using a variety of diffusion-weighted magnetic resonance pulse sequences. During inspiration breath-hold, the gas diffuses within the lung microstructure and the "apparent" diffusion coefficient (ADC) is derived such that it reflects the level of restriction of the gas to diffusion, thereby providing in vivo measurements of the distal airspaces [39, 40]. In addition to lung microstructural information, ventilation images may be acquired that show the regional distribution of inhaled gas [41-46]. ${ }^{3} \mathrm{He}$ static ventilation images and diffusion-weighted images are shown in figure 3 for a single healthy volunteer and two COPD patients in whom ${ }^{3} \mathrm{He}$ ventilation defects are readily visualised. The ${ }^{3} \mathrm{He}$ ADC maps are also brighter, reflecting larger ADC numbers for subjects with COPD that are indicative of pathological enlargement of the distal airspaces.

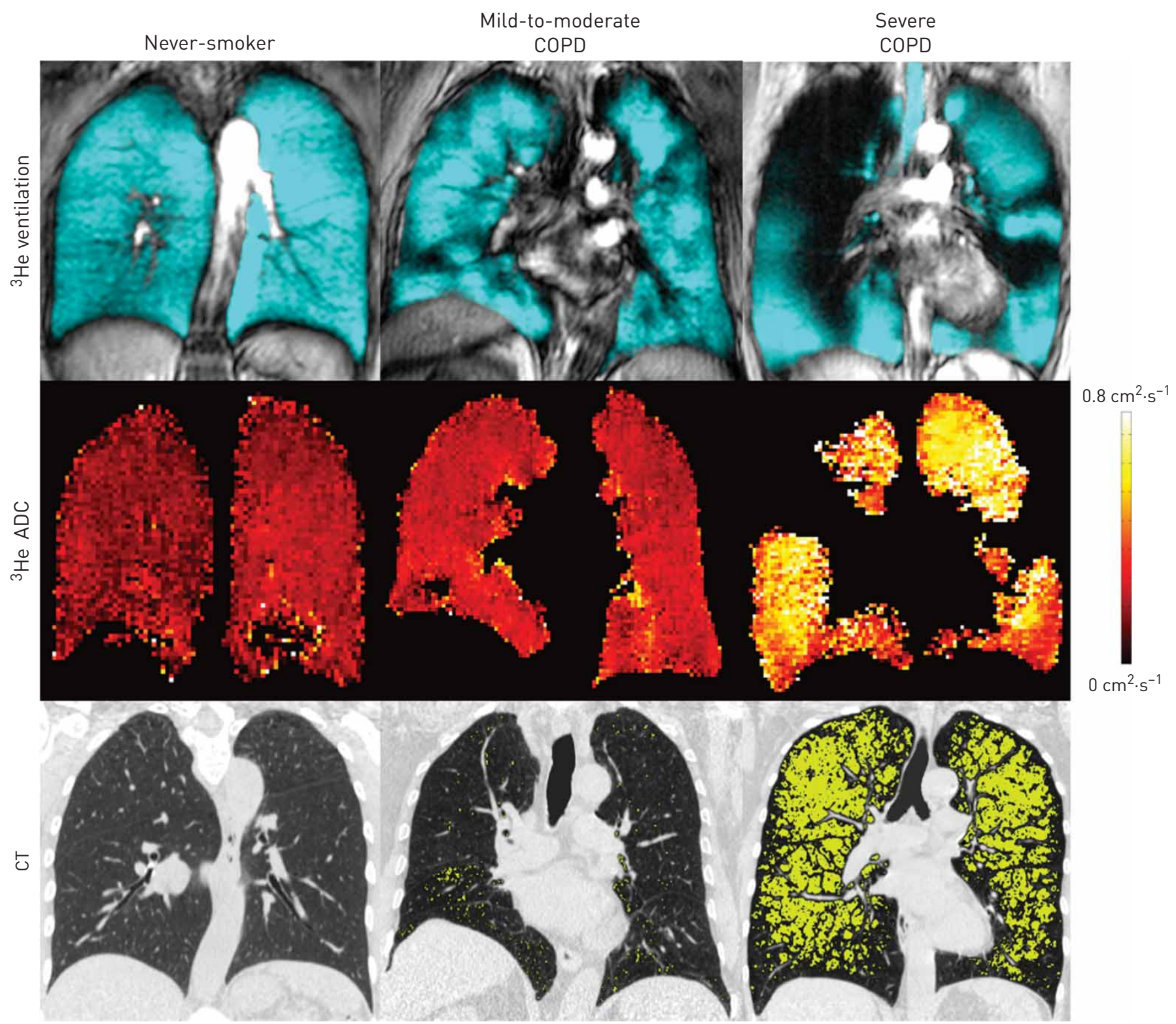

FIGURE 3 Pulmonary magnetic resonance imaging (MRI) and computed tomography (CT) in an older never-smoker and two ex-smokers. COPD: chronic obstructive pulmonary disease; ADC: "apparent" diffusion coefficient; VDP: ventilation defect percent; RA950: relative area of the CT density histogram voxels < $-950 \mathrm{HU}$; see figure 2 for definitions of pulmonary function and other tests. Never-smoker: 64-year-old female, FEV 1 $124 \%$ predicted, FEV 1 FVC $85 \%$, RV/TLC $73 \%$ predicted, DLC0 $78 \%$ predicted, 6MWD not performed, pack-years <0.5, MRI VDP 1\%, MRI ADC $0.22 \mathrm{~cm}^{2} \cdot \mathrm{s}^{-1}$. RA950 0\%. Mild-to-moderate COPD: 76-year-old male, FEV $177 \%$ predicted, FEV1/FVC 57\%, RV/TLC 117\% predicted, DLCo 70\% predicted, 6MWD $357 \mathrm{~m}$, pack-years 72, MRI VDP 7\%, MRI ADC $0.30 \mathrm{~cm}^{2} \cdot \mathrm{s}^{-1}$, RA950 2\%. Severe COPD: 69-year-old female, FEV1 33\% predicted, FEV $1 /$ FVC $39 \%$, RV/TLC $166 \%$ predicted, DLCo $28 \%$ predicted, 6MWD $366 \mathrm{~m}$, pack-years 106, MRI VDP $34 \%, \mathrm{MRI}^{\mathrm{A} D C} 0.51 \mathrm{~cm}^{2} \cdot \mathrm{s}^{-1}, \mathrm{RA} 95033 \%$. 


\section{Perfusion}

First-pass perfusion contrast-enhanced MRI involves an i.v. bolus injection of a gadolinium chelate contrast agent during continuous time-resolved T1-weighted ultrashort repetition time and echo time gradient echo imaging. The resulting images can be assessed visually or objectively to detect and quantify relative differences in immediate and delayed regional enhancement that represent perfusion defects [47]. Absolute quantification, however, remains difficult due to the nonlinear relationship of contrast material concentration and T1-shortening effects [48, 49].

Additional assessments of regional lung perfusion can be obtained with the administration of inhaled agents such as hyperpolarised ${ }^{129} \mathrm{Xe}$. Hyperpolarised ${ }^{129} \mathrm{Xe}$ gas is soluble in blood and tissues, making it possible to simultaneously measure regional perfusion [50] and alveolar transport kinetics [51]. While there is still limited clinical experience using this approach in COPD patients, measurements have been shown to be strongly associated with the diffusing capacity of the lung for carbon monoxide in patients with idiopathic pulmonary fibrosis [52].

The sine qua non for all such approaches is their validation against other outcomes so these may be considered in European Medicines Agency and US Food and Drug Administration COPD drug and device therapy regulatory approval processes. Moreover, for reimbursement through national formularies, cost-effectiveness and healthcare cost savings need to be considered, which is especially important if we consider novel complex technologies that are more commonly based in hospital radiology departments. In this regard, advanced MRI methods are poised for implementation at a number of sites in the USA and Canada, as well as in the European Union. For example, ${ }^{129} \mathrm{Xe}$ MRI is already approved for clinical use in the UK (National Health Service) and clinical approval in the USA is currently under development.

\section{What is next?}

Given the recent research investments in pulmonary imaging for COPD and the myriad of approaches available, thoracic CT and MRI, either independently or in combination, can provide detailed in vivo measurements of the parenchyma, airways and vasculature as well as more holistic assessments of the comorbid conditions present in COPD patients. Why then have they not revolutionised therapy and led to a cure? We believe that the answer to this question can be found in both the features being examined and how they are utilised (technology costs and complexity as well as choice of patients) in clinical investigations.

Pulmonary imaging in clinical, epidemiological and genetic investigation has been most heavily utilised for correlative analyses. How does the degree of parenchymal remodelling on CT relate to symptomatic or functional limitations found in a cohort of smokers? Do patients with more severe expiratory airflow obstruction have greater defects in the perfusion of the peripheral parenchyma?

Such studies of people with a shared exposure to tobacco smoke who represent a spectrum of disease severity have told us what we already suspect: sicker people tend to look worse by any measure. An airway wall may be fractionally thicker or a perfusion defect relatively more striking, but we do not have absolutes because we have not defined what is normal. Furthermore, for some of these features, the knowledge of what is normal may not enhance their utility in clinical care.

The expectations placed on imaging in clinical care are higher than those in biomedical research. What then is a potential path forward? We think two key approaches may help: 1) development of imaging biomarkers of lung injury in those at risk for future disease, and 2) utilisation of novel imaging phenotypes in patients with advanced disease to optimise care and to stratify patients in clinical trials of new therapies. We summarise our proposed approach in table 3 and provide a more detailed description in the following sections.

\section{Biomarkers of early COPD}

As shown in figure 4, MRI can provide exquisite in vivo assessments of alveolar microstructure and regional defects in ventilation even in ex-smokers at the very earliest stages of disease when they present without airflow limitation or CT evidence of airways disease or emphysema [53]. With very little additional effort, we think it is possible and quite necessary to define normal age- and sex-based distributions of ADC and regional lung ventilation. Such measurements could then be applied in focused assessments of those at greatest risk of future lung disease, not the 60-year-old 40 pack-year smoker with normal lung function who may never develop COPD, but the 25-year-old, 5 pack-year smoker reporting cough, wheeze or shortness of breath. Multiple birth cohort and long-term observational studies suggest that these latter symptomatic young adults may be at greatest risk for future airflow obstruction and parenchymal disease such as emphysema [54]. 
TABLE 3 What is next for chronic obstructive pulmonary disease (COPD) precision imaging?

\begin{tabular}{lcccc} 
& \multicolumn{4}{c}{ Time frame } \\
\cline { 2 - 5 } & $\begin{array}{c}\text { Now } \\
\text { (<1 year) }\end{array}$ & $\begin{array}{c}\text { Soon } \\
\text { (1 year) }\end{array}$ & $\begin{array}{c}\text { Later } \\
\text { (2-5 years) }\end{array}$ & $\begin{array}{c}\text { Long term } \\
\text { (10 years) }\end{array}$ \\
\hline $\begin{array}{l}\text { Early subclinical COPD phenotypes } \\
\text { Stratifying established COPD }\end{array}$ & $\checkmark$ & & & \\
$\begin{array}{l}\text { Machine and deep learning methods } \\
\text { Image-guided interventions }\end{array}$ & $\checkmark$ & $\checkmark$ & $\checkmark$ & $\checkmark$ \\
$\begin{array}{l}\text { Predict exacerbation risk } \\
\text { Explain symptoms/exercise capacity }\end{array}$ & $\checkmark$ & $\checkmark$ & & \\
$\begin{array}{l}\text { EMA/FDA approval of functional MRI } \\
\text { Use to change COPD outcomes }\end{array}$ & $\checkmark$ & & $\checkmark$ & $\checkmark$ \\
\hline
\end{tabular}

EMA: European Medicines Agency; FDA: US Food and Drug Administration; MRI: magnetic resonance imaging.

The first step to treating early and potentially reversible disease is to more dependently detect early disease. Further work is obviously needed to achieve this goal, but we need to be pragmatic in these efforts and not have the implementation of imaging based upon such parameters as the rate of decline in lung function. Longitudinal measures of function and physiology may be available in well-controlled investigations, but are not part of routine clinical care in young otherwise healthy individuals who may not even go for annual check-ups.

\section{Phenotyping established disease}

COPD is more than a spirometric abnormality and to understand how imaging may be impactful for patient care we must first establish a definition of disease. The true scope of such an effort is beyond this opinion piece, but there are practical criteria that can be immediately employed to enhance image utilisation. All patients with Global Initiative for Chronic Obstructive Lung Disease grade 3 and 4 COPD [55] should undergo CT imaging at least once to determine eligibility for resectional and nonresectional lung volume reduction. Additional disease criteria that can be used to guide the implementation of imaging include clinical impairment (symptoms and functional status) beyond what would be expected for disease stage, disease refractory to standard therapy and recurrent episodes of respiratory insufficiency/failure.

Imaging may reveal bronchiectatic dilation of the airways, right ventricular enlargement, coronary calcification or sarcopenia. Each represents a unique condition not discernible by measures of lung function, and the knowledge of their presence could significantly impact further clinical evaluation and therapeutic intervention. Even in the absence of such comorbid conditions, imaging may prove to be a useful predictor of healthcare utilisation. Examples of this include a recent investigation where ${ }^{3} \mathrm{He}$ MRI measurements, but not lung function, were significant predictors of hospitalisation in patients with mild-to-moderate COPD [56].

We acknowledge that some of our criteria for imaging utilisation, such as disproportionate symptoms and the presence of refractory disease, are difficult to objectify, but imaging is meant to augment and not replace clinical acumen. These "softer" indications force a necessary engagement between clinicians and the imaging community, whereas up to now efforts have been largely siloed and often lacked guidance from experts in bedside medicine. Finally, we must also contend with the increasing utilisation of CT scanning as a screening tool for the early detection of lung cancer. COPD is largely undiagnosed in the community and treatment is predicated on awareness of a condition. The presence of emphysema or other comorbid conditions provides a point of entry for these patients into the healthcare system where additional diagnostics can be performed and management plans can be instituted.

\section{Looking forward: the potential for deep learning and artificial intelligence}

The field of medical image analysis is undergoing rapid changes that mirror the improvements made in computational speed and capacity. The potential provided by artificial intelligence methods to analyse images has, to some extent, outstripped our ability to clinically leverage the information they provide. New approaches to data extraction are now possible and the research community is now also changing how images are quantified and processed. No longer are we constrained to measuring anatomical or physiological features of interest to predict clinical outcomes. We can now use techniques such as convolutional neural networks to mine the information content in medical images and predict outcomes without measuring the visible and sometimes less visible features responsible. We may similarly wish to 


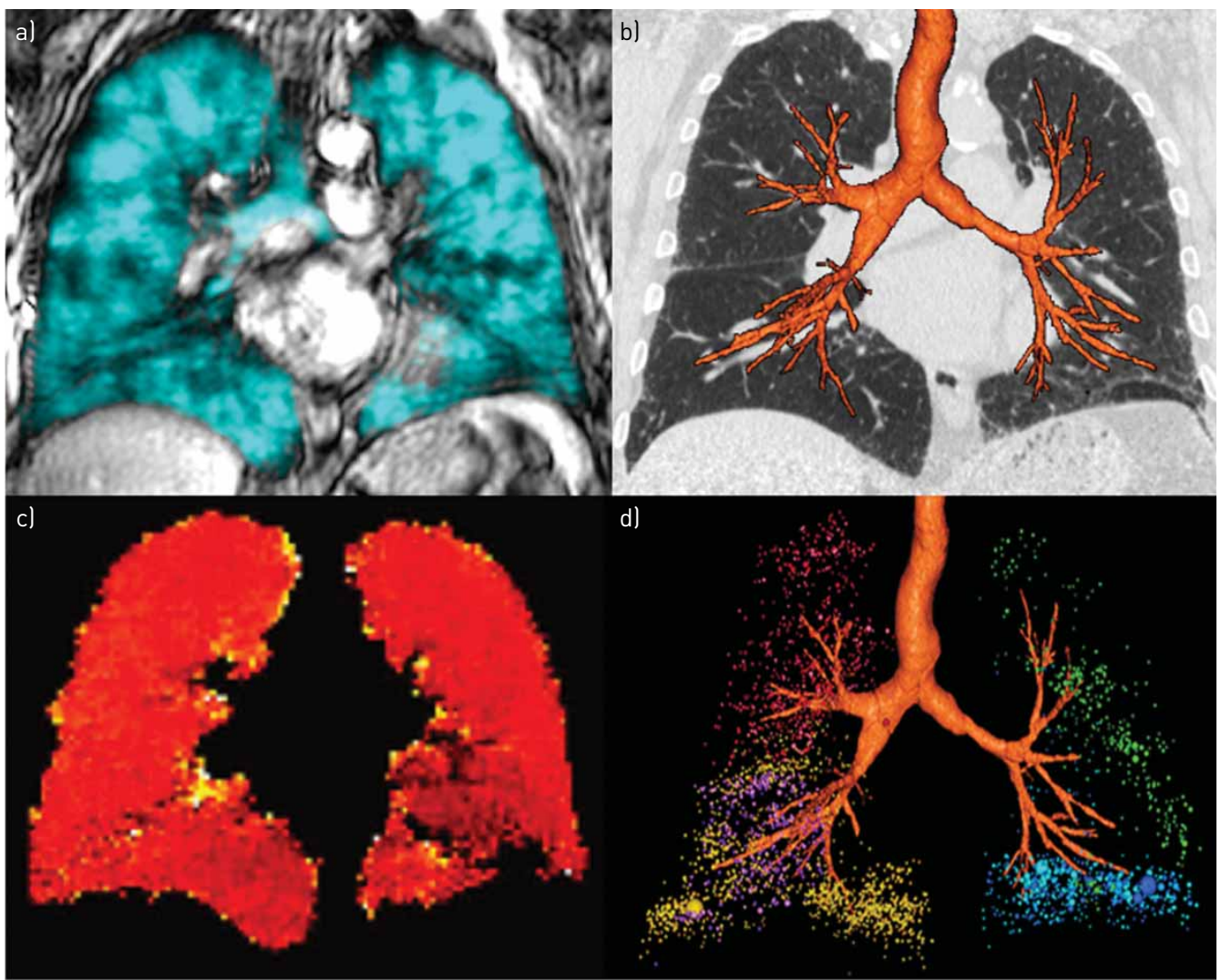

$0 \mathrm{~cm}^{2} \cdot \mathrm{s}^{-1}$ $0.8 \mathrm{~cm}^{2} \cdot \mathrm{s}^{-1}$

FIGURE 4 Ex-smoker without computed tomography (CT) or spirometric evidence of chronic obstructive pulmonary disease and low diffusing capacity of the lung for carbon monoxide (DLCO). ADC: "apparent" diffusion coefficient; VDP: ventilation defect percent; RA950: relative area of the CT density histogram voxels $<-950 \mathrm{HU}$; LAC: low-attenuation $(<-950 \mathrm{HU}$ ) clusters; see figures 2 and 3 for definitions of pulmonary function and other tests. a) ${ }^{3} \mathrm{He}$ ventilation, b) CT with airway tree reconstruction, c) ${ }^{3} \mathrm{He}$ ADC, and d) CT airway tree and LACs coloured by lobe. Ex-smoker: 74-year-old male, FEV1 89\% predicted, FEV1/FVC 77\%, RV/TLC $109 \%$ predicted, DLCo $41 \%$ predicted, 6MWD $299 \mathrm{~m}$, pack-years 50, MRI VDP 5\%, MRI ADC $0.31 \mathrm{~cm}^{2} \cdot \mathrm{s}^{-1}$, RA950 $2 \%$, LAC -2.1 .

predict future acute respiratory exacerbations, hospitalisations or other forms of healthcare utilisation in COPD patients undergoing a clinically indicated CT scan or otherwise "normal undiagnosed" former smokers undergoing lung cancer screening. Now that deep learning methods provide a way to reveal novel predictive information based on patient images, it is important to safeguard and not discard clinically actionable data and features that were previously hidden within COPD images.

\section{Conclusions}

We are in the early stages of understanding of how MRI and CT may be used to personalise and optimise therapy in patients with COPD. Until now, we have not exploited the pathological information provided by these imaging tools to stratify patients in clinical trials nor used imaging phenotypes to drive the development of new therapies or guide treatment decisions in complex COPD patients. We suggest that our current "one size fits all" approach based on spirometry information needs to change, and we must embrace higher-risk research and development strategies in COPD. Bold ideas and proposals need to be explored about all the different pathologies that reside within the COPD umbrella term and reclassifying COPD patient severity based on imaging measurements of the pathologies directly responsible for symptoms and progression. Renaming these different phenotypes in COPD, especially in early stages of the disease, may comprise a first step towards developing and using precision imaging to generate a deeper understanding of disease overlap/co-occurrence and the initiation of personalised or, at least, optimised therapies.

Acknowledgements: We thank our co-workers and trainees for their insights and collaboration, and the study participants who have stimulated the ideas and opinions expressed in this article, as well as A.L. Barker (Dept of Medical Biophysics, Western University, London, ON, Canada) and A. Diaz (Brigham and Women's Hospital, Boston, 
MA, USA) for figure 1 and R.L. Eddy (Dept of Medical Biophysics, Western University, London, ON, Canada) for figures $2-4$.

Conflict of interest: None declared.

Support statement: G. Parraga was supported by a Faculty Scholar award from Western University and salary support from the Canadian Institutes of Health Research (CIHR) New Investigator Award.

\section{References}

$1 \quad$ Fletcher C, Peto R. The natural history of chronic airflow obstruction. Br Med J 1977; 1: 1645-1648.

2 Anthonisen NR, Skeans MA, Wise RA, et al. The effects of a smoking cessation intervention on 14.5-year mortality: a randomized clinical trial. Ann Intern Med 2005; 142: 233-239.

3 Fishman A, Martinez F, Naunheim K, et al. A randomized trial comparing lung-volume-reduction surgery with medical therapy for severe emphysema. N Engl J Med 2003; 348: 2059-2073.

4 Nocturnal Oxygen Therapy Trial Group. Continuous or nocturnal oxygen therapy in hypoxemic chronic obstructive lung disease: a clinical trial. Ann Intern Med 1980; 93: 391-398.

5 Medical Research Council Working Party. Long term domiciliary oxygen therapy in chronic hypoxic cor pulmonale complicating chronic bronchitis and emphysema. Lancet 1981; 1: 681-686.

6 O’Donnell DE, Lam M, Webb KA. Spirometric correlates of improvement in exercise performance after anticholinergic therapy in chronic obstructive pulmonary disease. Am J Respir Crit Care Med 1999; 160: 542-549.

7 Fabbri LM, Calverley PM, Izquierdo-Alonso JL, et al. Roflumilast in moderate-to-severe chronic obstructive pulmonary disease treated with longacting bronchodilators: two randomised clinical trials. Lancet 2009; 374: 695-703.

8 Calverley PM, Rabe KF, Goehring UM, et al. Roflumilast in symptomatic chronic obstructive pulmonary disease: two randomised clinical trials. Lancet 2009; 374: 685-694.

9 Centers for Disease Control and Prevention. Chronic Obstructive Pulmonary Disease Surveillance - United States, 1971-2000. MMWR Surveill Summ 2002; 51 (6): 1-16.

10 Leopold JG, Gough J. The centrilobular form of hypertrophic emphysema and its relation to chronic bronchitis. Thorax 1957; 12: 219-235.

11 Muller NL, Staples CA, Miller RR, et al. "Density mask". An objective method to quantitate emphysema using computed tomography. Chest 1988; 94: 782-787.

12 Gevenois PA, De Vuyst P, de Maertelaer V, et al. Comparison of computed density and microscopic morphometry in pulmonary emphysema. Am J Respir Crit Care Med 1996; 154: 187-192.

13 Gevenois PA, de Maertelaer V, De Vuyst P, et al. Comparison of computed density and macroscopic morphometry in pulmonary emphysema. Am J Respir Crit Care Med 1995; 152: 653-657.

14 Dirksen A, Friis M, Olesen KP, et al. Progress of emphysema in severe alpha 1-antitrypsin deficiency as assessed by annual CT. Acta Radiol 1997; 38: 826-832.

15 Sciurba FC, Ernst A, Herth FJ, et al. A randomized study of endobronchial valves for advanced emphysema. N Engl J Med 2010; 363: 1233-1244.

16 Han MK, Kazerooni EA, Lynch DA, et al. Chronic obstructive pulmonary disease exacerbations in the COPDGene study: associated radiologic phenotypes. Radiology 2011; 261: 274-282.

17 Smith BM, Traboulsi H, Austin JHM, et al. Human airway branch variation and chronic obstructive pulmonary disease. Proc Natl Acad Sci USA 2018; 115: E974-E981.

18 Woodruff PG, Couper D, Han MK. Symptoms in smokers with preserved pulmonary function. N Engl J Med 2016; 375: 896-897.

19 Matsuoka S, Kurihara Y, Yagihashi K, et al. Quantitative assessment of air trapping in chronic obstructive pulmonary disease using inspiratory and expiratory volumetric MDCT. AJR Am J Roentgenol 2008; 190: 762-769.

20 O'Donnell RA, Peebles C, Ward JA, et al. Relationship between peripheral airway dysfunction, airway obstruction, and neutrophilic inflammation in COPD. Thorax 2004; 59: 837-842.

21 Eda S, Kubo K, Fujimoto K, et al. The relations between expiratory chest CT using helical CT and pulmonary function tests in emphysema. Am J Respir Crit Care Med 1997; 155: 1290-1294.

22 Bodduluri S, Bhatt SP, Hoffman EA, et al. Biomechanical CT metrics are associated with patient outcomes in COPD. Thorax 2017; 72: 409-414.

23 Galban CJ, Han MK, Boes JL, et al. Computed tomography-based biomarker provides unique signature for diagnosis of COPD phenotypes and disease progression. Nat Med 2012; 18: 1711-1715.

24 Kim EY, Seo JB, Lee HJ, et al. Detailed analysis of the density change on chest CT of COPD using non-rigid registration of inspiration/expiration CT scans. Eur Radiol 2015; 25: 541-549.

25 Boes JL, Hoff BA, Bule M, et al. Parametric response mapping monitors temporal changes on lung CT scans in the Subpopulations and Intermediate Outcome Measures in COPD Study (SPIROMICS). Acad Radiol 2015; 22: 186-194.

26 Capaldi DP, Zha N, Guo F, et al. Pulmonary imaging biomarkers of gas trapping and emphysema in COPD: ${ }^{3} \mathrm{He}$ MR imaging and CT parametric response maps. Radiology 2016; 279: 597-608.

27 Bhatt SP, Soler X, Wang X, et al. Association between functional small airways disease and FEV decline in COPD. Am J Respir Crit Care Med 2016; 15: 178-184.

28 Estepar RS, Kinney GL, Black-Shinn JL, et al. Computed tomographic measures of pulmonary vascular morphology in smokers and their clinical implications. Am J Respir Crit Care Med 2013; 188: 231-239.

29 Rahaghi FN, Vegas-Sanchez-Ferrero G, Minhas JK, et al. Ventricular geometry from non-contrast non-ECG-gated CT scans: an imaging marker of cardiopulmonary disease in smokers. Acad Radiol 2017; 24: 594-602.

30 Iyer KS, Newell JD Jr, Jin D, et al. Quantitative dual-energy computed tomography supports a vascular etiology of smoking-induced inflammatory lung disease. Am J Respir Crit Care Med 2016; 193: 652-661.

31 Bhatt SP, Vegas-Sanchez-Ferrero G, Rahaghi FN, et al. Cardiac morphometry on computed tomography and exacerbation reduction with beta-blocker therapy in chronic obstructive pulmonary disease. Am J Respir Crit Care Med 2017; 196: 1484-1488.

32 Chae EJ, Seo JB, Goo HW, et al. Xenon ventilation CT with a dual-energy technique of dual-source CT: initial experience. Radiology 2008; 248: 615-624. 
33 Lee CW, Seo JB, Lee Y, et al. A pilot trial on pulmonary emphysema quantification and perfusion mapping in a single-step using contrast-enhanced dual-energy computed tomography. Invest Radiol 2012; 47: 92-97.

34 Park EA, Goo JM, Park SJ, et al. Chronic obstructive pulmonary disease: quantitative and visual ventilation pattern analysis at xenon ventilation CT performed by using a dual-energy technique. Radiology 2010; 256: 985-997.

35 Hwang HJ, Seo JB, Lee SM, et al. Assessment of regional xenon ventilation, perfusion, and ventilation-perfusion mismatch using dual-energy computed tomography in chronic obstructive pulmonary disease patients. Invest Radiol 2016; 51: 306-315.

36 Lutey BA, Lefrak SS, Woods JC, et al. Hyperpolarized ${ }^{3} \mathrm{He}$ MR imaging: physiologic monitoring observations and safety considerations in 100 consecutive subjects. Radiology 2008; 248: 655-661.

37 Shukla Y, Wheatley A, Kirby M, et al. Hyperpolarized ${ }^{129}$ Xe magnetic resonance imaging: tolerability in healthy volunteers and subjects with pulmonary disease. Acad Radiol 2012; 19: 941-951.

38 Albert MS, Cates GD, Driehuys B, et al. Biological magnetic resonance imaging using laser-polarized ${ }^{129}$ Xe. Nature 1994; 370: 199-201.

39 Woods JC, Choong CK, Yablonskiy DA, et al. Hyperpolarized ${ }^{3} \mathrm{He}$ diffusion MRI and histology in pulmonary emphysema. Magn Reson Med 2006; 56: 1293-1300.

40 Thomen RP, Quirk JD, Roach D, et al. Direct comparison of ${ }^{129}$ Xe diffusion measurements with quantitative histology in human lungs. Magn Reson Med 2017; 77: 265-272.

41 Parraga G, Ouriadov A, Evans A, et al. Hyperpolarized ${ }^{3} \mathrm{He}$ ventilation defects and apparent diffusion coefficients in chronic obstructive pulmonary disease: preliminary results at 3.0 Tesla. Invest Radiol 2007; 42: 384-391.

42 Samee S, Altes T, Powers P, et al. Imaging the lungs in asthmatic patients by using hyperpolarized helium-3 magnetic resonance: assessment of response to methacholine and exercise challenge. J Allergy Clin Immunol 2003; 111: 1205-1211.

43 Kauczor HU, Markstaller K, Puderbach M, et al. Volumetry of ventilated airspaces by ${ }^{3} \mathrm{He}$ MRI: preliminary results. Invest Radiol 2001; 36: 110-114.

44 Tustison NJ, Altes TA, Song G, et al. Feature analysis of hyperpolarized helium-3 pulmonary MRI: a study of asthmatics versus nonasthmatics. Magn Reson Med 2010; 63: 1448-1455.

45 Kirby M, Heydarian M, Svenningsen S, et al. Hyperpolarized He-3 magnetic resonance functional imaging semiautomated segmentation. Acad Radiol 2012; 19: 141-152.

46 Guo F, Yuan J, Rajchl M, et al. Globally optimal co-segmentation of three-dimensional pulmonary ${ }^{1} \mathrm{H}$ and hyperpolarized ${ }^{3} \mathrm{He}$ MRI with spatial consistence prior. Med Image Anal 2015; 23: 43-55.

47 Risse F, Eichinger M, Kauczor HU, et al. Improved visualization of delayed perfusion in lung MRI. Eur J Radiol 2011; 77: 105-110.

48 Puderbach M, Risse F, Biederer J, et al. In vivo Gd-DTPA concentration for MR lung perfusion measurements: assessment with computed tomography in a porcine model. Eur Radiol 2008; 18: 2102-2107.

49 Ohno Y, Murase K, Higashino T, et al. Assessment of bolus injection protocol with appropriate concentration for quantitative assessment of pulmonary perfusion by dynamic contrast-enhanced MR imaging. J Magn Reson Imaging 2007; 25: 55-65.

50 Cleveland ZI, Cofer GP, Metz G, et al. Hyperpolarized ${ }^{129}$ Xe MR imaging of alveolar gas uptake in humans. PLoS One 2010; 5: e12192.

51 Patz S, Hersman FW, Muradian I, et al. Hyperpolarized ${ }^{129}$ Xe MRI: a viable functional lung imaging modality? Eur J Radiol 2007; 64: 335-344.

52 Kaushik SS, Freeman MS, Yoon SW, et al. Measuring diffusion limitation with a perfusion-limited gas hyperpolarized ${ }^{129} \mathrm{Xe}$ gas-transfer spectroscopy in patients with idiopathic pulmonary fibrosis. J Appl Physiol 2014; 117: 577-585.

53 Kirby M, Owrangi A, Svenningsen S, et al. On the role of abnormal $\mathrm{DL}_{\mathrm{CO}}$ in ex-smokers without airflow limitation: symptoms, exercise capacity and hyperpolarised helium-3 MRI. Thorax 2013; 68: 752-759.

54 Hayden LP, Hardin ME, Qiu W, et al. Asthma is a risk factor for respiratory exacerbations without increased rate of lung function decline: five-year follow-up in adult smokers from the COPDGene study. Chest 2018; 153: 368-377.

55 Global Initiative for Chronic Obstructive Lung Disease. Global Strategy for the Diagnosis, Management and Prevention of Chronic Obstructive Pulmonary Disease. 2017. http://goldcopd.org/gold-2017-global-strategydiagnosis-management-prevention-copd Date last accessed: October 5, 2018

56 Kirby $\mathrm{M}$, Pike D, Coxson $\mathrm{HO}$, et al. Hyperpolarized ${ }^{3} \mathrm{He}$ ventilation defects used to predict pulmonary exacerbations in mild to moderate chronic obstructive pulmonary disease. Radiology 2014; 273: 887-896. 